\title{
The Potential Clinical Implications of Telomerase Reverse Transcriptase in the Detection and Diagnosis of Bladder Cancer
}

\author{
Yitian Wang ${ }^{1}$, Bo Wang ${ }^{1}$, Meng Zhang ${ }^{1}$, Zhiming Cai ${ }^{1^{*}}$ and Song $\mathbf{W u}^{1,2^{*}}$ \\ ${ }^{1}$ Shenzhen Second People's Hospital, clinical medicine college of Anhui Medical University, Shenzhen Guangdong, China \\ 2Zhongshan School of Medicine, Sun Yat-sen University, Guangzhou Guangdong, China
}

*Corresponding authors: Song Wu, Shenzhen Second People's Hospital, clinical medicine college of Anhui Medical University, Shenzhen Guangdong, China, Tel: +0086010075583669030; Fax: +0086010075583669030; E-mail: Doctor_Wusong@126.com

Zhiming Cai, Shenzhen Second People's Hospital, clinical medicine college of Anhui Medical University, Shenzhen Guangdong, China, Tel: +0086010075583669030; Fax: +0086010075583669030; E-mail: 547349961@qq.com

Rec date: Apr 21, 2014, Acc date: Jun 25, 2014, Pub date: Jun 27, 2014

Copyright: (c) 2014 Wang $Y$ et al. This is an open-access article distributed under the terms of the Creative Commons Attribution License, which permits unrestricted use, distribution, and reproduction in any medium, provided the original author and source are credited

\begin{abstract}
Telomerase and the control of telomere length are intimately linked to the process of tumorigenesis in humans and mutations in the promoter regions of its catalytic subunit, TERT, can affect telomerase activity and telomere, creating severe clinical phenotypes. In this paper, we briefly review the potential mechanisms under which TERT play in tumorigenesis and focus mainly on TERT's potential as a biomarker for the detection and diagnosis of bladder cancer.
\end{abstract}

Keywords: Telomere; Telomerase reverse transcriptase; Bladder cancer

\section{Introduction}

Telomeres are the structures that cap the ends of chromosomes. They are composed of the hexameric DNA sequence TTAGGG tandemly repeated into arrays of $25 \mathrm{~kb}$ in our cells. One important function of telomeres is to counteract the end-replication losses that occur resulting from semiconservative replication of DNA molecules [1]. And the telomerase reverse transcriptase (TERT), along with the telomerase RNA component (TERC), regulates the above-mentioned function by catalysing the RNA-templated addition of telomere repeats at the chromosomal terminus [2]. It is noted that, more than $90 \%$ of human cancers are telomerase positive, whereas most normal tissues or benign tumors contain low or undetectable telomerase activity [3]. In particular, the enhanced levels of the hTERT catalytic subunit have been observed in nearly all human tumor histotypes [4]. While recent findings provided the description of TERT promoter mutations in $66 \%$ of muscle-invasive urothelial carcinomas of the bladder [5] the detection of TERT promoter mutations in urine holds promise in the diagnosis of bladder cancers [6]. Besides, studies also demonstrated the close relationship between elevated TERT expression and the pathological grades and clinical stages of bladder cancers [7]. Nevertheless, the potential mechanism under which TERT mutations perform in tumorigenesis and its clinical implications in bladder cancer still need to be elucidated. Hence, the purpose of this review is to highlight the available evidence, summarize the potential roles TERT has in tumorigenesis and put forward the potential clinical implications and applications in bladder cancers.

\section{Potential mechanism under which TERT contributes to the development of carcinoma}

The expression of hTERT correlates with telomerase activity because its presence is essential for enzymatic activity, and researches have indicated that telomerase activation is viewed as one of the six key events common to cancer development [8]. While telomere maintenance is one feasible mechanism for tumorigenesis and cancer progression, increasing evidence suggests that there are other two potential ones [7]. Firstly, as studies have suggested that some immortalized cells were found to be telomerase negative, [9] researchers have put forward the corresponding alternative lengthening of telomeres (ALT) theory after excluding the possible technical reasons [10]. Secondly, the finding that TERT is involved in the regulation of Wnt target genes may offer mechanistic evidence for a telomere-independant function of TERT in tumorigenesis and cancer development [11]. Taken together, TERT plays a vital position in tumorigenesis and cancer development, but the pathways and molecular mechanisms by which TERT promotes cancer still needs to be elucidated.

\section{The application of TERT in the detection and diagnosis of bladder cancer}

Telomerase activity and hTERT are found in nearly $90 \%$ of bladder cancers [12]. Nevertheless, their presence is not specific for malignancy. Other studies have found that the elevated hTERT expression is associated with pathological grade and clinical stage of cancer. In clinical practice, testing for telomerase in exfoliated cells collected from bladder washings can be a promising tool for the diagnosis and management of bladder cancer. However, other studies have indicated that telomerase activity in urine is not related to tumor grade, size or stage due to the rate of false-positive results. As an alternative, the assay of hTERT expression in urine can be a more sensitive test for the detection of primary urothelial neoplasms than do assessments for telomerase activity. Recently, the role of TERT in the detection and diagnosis of bladder cancer has been widely reviewed. The potential relevance of TERT for diagnosing this malignancy derives from 2 main findings. TERT promoter mutations and TERT mRNA expression have been detected in most cases of transitional cell carcinoma. 
Page 2 of 3

According to recent studies, frequent TERT promoter mutations were found in various types of cancers but not in adjacent normal tissues [13]. One of the promising results was finding point mutations in the TERT gene promoter in $66 \%$ of urothelial carcinomas of the bladder [13]. In one study, researchers sequenced the TERT promoter in 76 well-characterized papillary and flat noninvasive urothelial carcinomas, which demonstrated the early occurrence of TERT promoter mutations in urothelial neoplasia [5]. In another study, people investigated a large series of 327 patients with wellcharacterized tumors from urothelial cell carcinoma of bladder for mutations in the TERT promoter and they observed that the common TERT promoter mutations affected two-thirds of the tumors and were spread across all stages and grades, suggesting that the mutations are early events in bladder carcinogenesis [14]. And the subsequent research led to the findings which suggest that the TERT promoter mutations in conjunction with the common polymorphism have potential of being used as clinical biomarkers in bladder cancer. Then researchers tried to test the sensitivity of urine TERT promoter mutation detection, subsequently, in one study, fifty-one mutations were detected including all mutations $(n=23)$ in 29 patients with lowgrade non-invasive tumours, only two mutations detected in tumour samples were not detected in the corresponding urine, possibly due to the presence of a low number of tumour cells [15]. Taken together, these provocative results suggest that TERT promoter mutations occur in both papillary and flat lesions, are the most frequent genetic alterations identified to date in noninvasive precursor lesions of the bladder, and may offer a useful urinary biomarker for both early detection and monitoring of bladder neoplasia.

On the other side, as telomerase activity in cancer exfoliated cells was not correlated with the clinical stage and grade, [16] detecting hTERT mRNA by RT-PCR is developed with a much better sensitivity than the detection of telomerase activity when adopted in diagnosing bladder cancers. When adopting a quantitative RT-PCR assay, researchers showed that the levels of hTERT mRNA expression are significantly correlated with bladder cancer grade and stage [12]. Others have also showed that the combination of hTERT and cytology can largely increase the sensitivity of cytology [17]. Although researches have suggested that quantitative hTR analysis is the most accurate telomerase-based test for bladder cancer detection, [18] they are unable to exclude the problem of interference of leukocytes with urine-based tests. As an alternative, individuals can be submitted to hTERT testing, which keeps its specificity in the presence of leukocyturia.

To summarize, hTERT mRNA detection by real-time quantitative RT-PCR and TERT promoter mutations detection in urine both appear to be sensitive methods for the detection and diagnosis of bladder cancers.

\section{Conclusion}

TERT activity has been measured in a wide variety of cancerous and non-cancerous tissues, and the vast majority of studies have shown the association between TERT activity and the presence of cancerous cells. Regarding TERT's contribution to tumorigenesis, telomere maintenance, alternative lengthening of telomere and telomereindependent ways are the three common ones known to us. In bladder cancers, the detection of TERT mRNA and TERT promoter mutations in urine also enable it to be a promising biomarker for patients' diagnosis when comparing with the existing ones. Nevertheless, those ideas might be associated with several problems, which still need to be improved and developed.

\section{Acknowledgements}

The work by Zhiming Cai and Song Wu was supported by Natural Science Foundation of China 81301740, as well as the Shenzhen Second People's Hospital, clinical medicine college of Anhui Medical University, Zhongshan School of Medicine, Sun Yat-sen University.

\section{References}

1. Olovnikov AM (1971) [principle of marginotomy in template synthesis of polynucleotides]. Dokl Akad Nauk SSSR 201: 1496-1499.

2. Bodnar AG, Ouellette M, Frolkis M, Holt SE, Chiu CP, et al. (1998) Extension of life-span by introduction of telomerase into normal human cells. Science 279: 349-352.

3. Kim NW, Piatyszek MA, Prowse KR, Harley CB, West MD, et al. (1994) Specific association of human telomerase activity with immortal cells and cancer. Science 266: 2011-2015.

4. Lantuejoul S, Soria JC, Moro-Sibilot D, Morat L, Veyrenc S, et al. (2004) Differential expression of telomerase reverse transcriptase (htert) in lung tumours. Br J cancer 90: 1222-1229.

5. Kinde I, Munari E, Faraj SF, Hruban RH, Schoenberg M, et al. (2013) Tert promoter mutations occur early in urothelial neoplasia and are biomarkers of early disease and disease recurrence in urine. Cancer Res 73: 7162-7167.

6. Allory Y, Beukers W, Sagrera A, Flandez M, Marques M, et al. (2014) Telomerase reverse transcriptase promoter mutations in bladder cancer: High frequency across stages, detection in urine, and lack of association with outcome. Euro urol 65: 360-366.

7. Martinez P, Blasco MA (2011) Telomeric and extra-telomeric roles for telomerase and the telomere-binding proteins. Nat Rev Cancer 11: 161-176.

8. Hanahan D, Weinberg RA (2011) Hallmarks of cancer: The next generation. Cell 144: 646-674.

9. Bryan TM, Englezou A, Gupta J, Bacchetti S, Reddel RR, et al. (1995) Telomere elongation in immortal human cells without detectable telomerase activity. EMBO 14: 4240-4248.

10. Bryan TM, Reddel RR (1997) Telomere dynamics and telomerase activity in in vitro immortalised human cells. Eur J cancer 33: 767-773.

11. Park JI, Venteicher AS, Hong JY, Choi J, Jun S, et al. (2009) Telomerase modulates wnt signalling by association with target gene chromatin. Nature 460: 66-72.

12. De Kok JB, Schalken JA, Aalders TW, Ruers TJ, Willems HL, et al. (2000) Quantitative measurement of telomerase reverse transcriptase (htert) mrna in urothelial cell carcinomas. Int J cancer 87: 217-220.

13. Killela PJ, Reitman ZJ, Jiao Y, Bettegowda C, Agrawal N, et al. (2013) Tert promoter mutations occur frequently in gliomas and a subset of tumors derived from cells with low rates of self-renewal. Pro Nat Acad Sci U S A 110: 6021-6026.

14. Rachakonda PS, Hosen I, de Verdier PJ, Fallah M, Heidenreich B, et al. (2013) Tert promoter mutations in bladder cancer affect patient survival and disease recurrence through modification by a common polymorphism. Pro Nat Acad Sci U S A 110: 17426-17431.

15. Hurst CD, Platt FM, Knowles MA (2014) Comprehensive mutation analysis of the tert promoter in bladder cancer and detection of mutations in voided urine. Euro urol 65: 367-369.

16. Gelmini S, Crisci A, Salvadori B, Pazzagli M, Selli C, et al. (2000) Comparison of telomerase activity in bladder carcinoma and exfoliated cells collected in urine and bladder washings, using a quantitative assay. Clin cancer res 6: 2771-2776.

17. Eissa S, Motawi T, Badr S, Zaghlool A, Maher A, et al. (2013) Evaluation of urinary human telomerase reverse transcriptase mrna and scatter 
Citation: Wang Y, Wang B, Zhang M, Cai Z, Wu S (2014) The Potential Clinical Implications of Telomerase Reverse Transcriptase in the Detection and Diagnosis of Bladder Cancer. Hereditary Genet 3: 131. doi:10.4172/2161-1041.1000131

Page 3 of 3

factor protein as urine markers for diagnosis of bladder cancer. Clini lab 59: 317-323.
18. Weikert S, Krause H, Wolff I, Christoph F, Schrader M, et al. (2005) Quantitative evaluation of telomerase subunits in urine as biomarkers for noninvasive detection of bladder cancer. Int J cancer 117: 274-280. 